\title{
Utilização de P-Index em uma bacia hidrográfica através de técnicas de geoprocessamento
}

\author{
Fabíola Lopes', Gustavo H. Merten², Melissa Franzen², Elvio Giasson', Fernanda Helfer ${ }^{2}$ \& Luis F. A. Cybis ${ }^{2}$
}

\section{RESUMO}

Devido aos grandes problemas de eutrofização de lagos e reservatórios, a identificação de áreas fornecedoras de fósforo possui grande importância no planejamento de medidas de controle da poluição hídrica por atividades agrícolas. Neste trabalho, aplicou-se uma metodologia denominada P-Index, para identificar áreas com potencial de fornecimento de fósforo de ecossistemas terrestres para os corpos d'água, cujo método está baseado no cruzamento de planos de informações referentes à disponibilidade de fósforo no solo e dos processos de transferência deste nutriente, para os cursos de água. Tal procedimento foi aplicado na bacia contribuinte do Sistema de Reservatório Salto, no município de São Francisco de Paula, RS. Com o cruzamento dos planos de informação, obteve-se um mapa de classes de contribuição de fósforo para os reservatórios, verificando-se que a quase totalidade desta área da bacia foi classificada como tendo baixo potencial de fornecimento. As pequenas áreas consideradas com maior potencial de contribuição representam áreas situadas próximas aos corpos d'água e sob cultivo de culturas como batata e alho, nos quais são aplicadas doses elevadas de fertilizantes.

Palavras-chave: eutrofização, uso da terra, Sistema de Informações Geográficas

\section{Use of P-Index in a waterbasin by geoprocessing techniques}

\begin{abstract}
Due to problems caused by eutrophication of lakes and reservoirs, the identification of phosphorus source areas is important for planning the control of agriculture-related water pollution. This study applied a method designed to identify these phosphorus source areas, called P-Index, which is based on combining data layers related to soil phosphorus availability and phosphorus transport processes. The method was used for studying the Salto Reservoir waterbasin, in São Francisco de Paula, in the State of Rio Grande do Sul, Brazil. Data related to phosphorus availability, intensity of transport processes, and distance to waterways were combined to yield a map of phosphorus contribution classes, which showed that almost the entire study area was considered to have low phosphorus contribution potential. A few small areas, corresponding to potato and garlic fields that received heavy chemical fertilization, were classified as having high phosphorus contribution potentials.
\end{abstract}

Key words: eutrofication, land use, Geographic Information System 


\section{INTRODUÇÃO}

O problema da eutrofização de lagos e reservatórios tem sido objeto de preocupação por parte da sociedade, em função dos impactos gerados na qualidade da água devido, principalmente, à possibilidade de ocorrência de cianobactérias tóxicas (Carmichael et al., 2001; Tundisi et al., 2004). A eutrofização dos corpos d'água está associada à presença de nutrientes, especialmente fósforo e nitrogênio, em concentrações acima dos níveis encontrados nas condições naturais (Yoo et al., 1995).

Na maioria das águas continentais, o fósforo é considerado o principal fator limitante da produtividade primária, não só porque é naturalmente escasso mas, também, da carga de fósforo ser facilmente consumida pelos corpos hídricos (Margalef, 1983). Por outro lado, o excesso de fósforo na forma dissolvida é, em geral, apontado como o principal responsável pela eutrofização artificial dos ecossistemas aquáticos.

O aparecimento de florações algais em reservatórios destinados à geração de energia elétrica e abastecimento vemse tornando cada vez mais comum, sobretudo naqueles situados próximo a áreas agrícolas.

De maneira geral, as atividades agrícolas contribuem, de forma substancial, para o fluxo de transferência de nutrientes para os corpos d'água. Nos Estados Unidos, por exemplo, admite-se que entre 50 a $60 \%$ da carga poluente que contamina os lagos e os rios, são provenientes da agricultura (Gburek \& Sharpley, 1998), enquanto na Europa se considera que entre 24 a $71 \%$ do total de fósforo transferido para os rios são originados de áreas agrícolas (Vighi \& Chiaudani, 1987).

As fontes de fósforo nas áreas agrícolas têm sua origem nos dejetos produzidos pelos sistemas que envolvem confinamento de animais e nas áreas de cultivo em que são aplicados fertilizantes (químico ou orgânico) em doses superiores àquelas que as plantas são capazes de absorver. A maior parte do fósforo removido das áreas agrícolas é transferida para os corpos d’água através do escoamento superficial, nas formas particulada e solúvel (Dils \& Heathwaite, 1996). A forma particulada é mais facilmente removível devido à característica do fósforo ser facilmente adsorvido por partículas de argila e óxidos do solo. A fração solúvel representa apenas uma pequena parcela do fósforo total transportado e assume maior importância onde seu transporte ocorre pelo escoamento subsuperficial não saturado (Dils \& Heathwaite, 1996).

A ocorrência dos processos erosivos e o transporte de sedimentos para os corpos d'água são os mecanismos básicos da transferência do fósforo das áreas agrícolas para os corpos d'água. Outro aspecto importante a ser considerado na transferência do fósforo, diz respeito à distância em que as áreas fontes de contaminação se encontram dos corpos d’água, sendo que as áreas mais próximas tendem a contribuir relativamente com maiores quantidades de fósforo.

Assim, em uma bacia hidrográfica as áreas que potencialmente poderiam contribuir para um aumento da concentração de fósforo nos ecossistemas aquáticos, seriam aque- las em que houvesse uma combinação de fatores, tais como: i) elevação dos teores de fósforo no solo; ii) aumento da susceptibilidade a erosão; iii) maior proximidade dos cursos de água. A possibilidade de identificar essas áreas passa a ser de enorme importância quando se deseja elaborar diagnósticos voltados ao planejamento de medidas destinadas ao controle de poluição hídrica por atividades agrícolas.

Uma possível alternativa a ser utilizada na identificação das áreas de risco consiste na elaboração de planos de informações que, uma vez sobrepostos, possam gerar, através de um ambiente SIG (Sistema de Informações Geográficas), um mapa de áreas de risco de contaminação com fósforo.

Com base nessas considerações, tem-se proposto uma maneira de identificar áreas de maior risco de contaminação com fósforo em bacias hidrográficas através de índices designadores de áreas de risco chamados P-Index (Lemunyon \& Gilbert, 1993; Eghball \& Gilley, 2001). A maioria dos P-Index leva em consideração, para a determinação de área de risco, fatores como áreas preferenciais de formação do escoamento, grau de erosão, quantidade de fósforo no solo, além das taxas e métodos de aplicação de fósforo no solo.

No Brasil não consta, na literatura, referência ao uso deste método para determinação de áreas de risco, porém problemas de florações de algas cianobactérias em lagos e reservatórios têm sido constatados com freqüência, sendo importante o estudo de ferramentas que sirvam como instrumentos para a gestão dos recursos hídricos. Nesta primeira aproximação, realizada através deste trabalho, tevese como propósito adaptar o uso do P-Index para a identificação de áreas de risco de contaminação de fósforo em um sistema de reservatórios em cascata, localizado na região Sul do Brasil.

\section{MATERIAL E MÉTODOS}

Este trabalho é parte integrante de um projeto de pesquisa que teve, como propósito principal, investigar as causas da ocorrência de uma floração de cianobactérias em um sistema de reservatórios em cascata (Franzen et al., 2004; Cybis, 2005). Para identificar as áreas que poderiam estar contribuindo para o fluxo de transferência de fósforo das bacias contribuintes para os reservatórios, utilizou-se um indicador de áreas de risco de fósforo do tipo P-Index.

O trabalho foi realizado na bacia hidrográfica dos reservatórios Divisa, Blang e Salto $\left(52,48 \mathrm{~km}^{2}\right)$, que se encontram na bacia hidrográfica do rio Caí, no estado do Rio Grande do Sul. Esses reservatórios integram o Sistema Salto de Hidrelétricas e foram construídos na década de cinqüenta para fins de geração de energia elétrica e regularização de vazões. A área da bacia encontra-se em uma região de clima subtropical úmido, sendo que, devido a grande precipitação pluviométrica, se verifica um excedente hídrico anual. O relevo da bacia é relativamente plano e levemente inclinado para os quadrantes de oeste. Nas áreas mais aplainadas predominam colinas côncavo-convexas com pequeno aprofundamento nos vales fluviais e presença de sulcos de erosão. A unidade amplamente representada na área de 
estudo pertence à formação Serra Geral, além de sedimentos recentes depositados principalmente ao longo das calhas dos rios. Conforme levantamento pedológico 1:750.000 (Brasil, 1973 apud Streck et al., 2002), atualizado segundo o Sistema Brasileiro de Classificação dos Solos - SBCS, a classe de solo predominante na região de estudo é Cambissolo Húmico típico associado com Neossolo Litólico. A vegetação nativa da região, composta de campos de Savanas e Matas de Araucária, vem sendo substituída por florestamento restrito a Pinus elliotis e Eucalyptus sp.

A composição do P-Index utilizada neste trabalho, refere-se a três fatores considerados fundamentais para determinação de áreas de vulnerabilidade de fósforo, conforme sugerem Lemunyon \& Gilbert (1993): i) perda de solo estimada; ii) distância entre a fonte de fósforo e o corpo d’água. e iii) teores de fósforo no solo.

Os valores medidos para esses fatores foram divididos em classes, conforme a Tabela 1.

Tabela 1. Fatores utilizados na composição do P-Index e suas respectivas classes

\begin{tabular}{lccccc}
\hline \multicolumn{1}{c}{ Fatores* $^{*}$} & \multicolumn{5}{c}{ Classes } \\
\cline { 2 - 6 } & $\begin{array}{c}\text { Nenhuma } \\
\mathbf{( 0 )}\end{array}$ & $\begin{array}{c}\text { Baixa } \\
\mathbf{( 1 )}\end{array}$ & $\begin{array}{c}\text { Média } \\
\mathbf{( 2 )}\end{array}$ & $\begin{array}{c}\text { Alta } \\
\mathbf{( 4 )}\end{array}$ & $\begin{array}{c}\text { M. Alta } \\
\mathbf{( 8 )}\end{array}$ \\
PS $\left(\mathrm{t} \mathrm{ha}^{-1}\right)$ & 0 & $0,1-0,5$ & $0,51-1,0$ & $1,1-1,5$ & $>1,5$ \\
DD $(\mathrm{m})$ & $>150$ & $150-100$ & $99-80$ & $79-50$ & $<50$ \\
NPS $\left(\mathrm{mg} \mathrm{kg}^{-1}\right)$ & $<1,1$ & $1,1-4,0$ & $4,1-6,0$ & $6,1-8,0$ & $>8,0$ \\
\hline
\end{tabular}

* PS - Perda de solo, DD - Distância da drenagem, NPS - Nível de fósforo no solo

Para obtenção do índice de risco de fósforo, os três fatores foram multiplicados no software Spring - INPE (Spring, 1996) e se obteve, então um valor numérico. A transformação do valor numérico em um índice de risco de fósforo foi determinada de acordo com a Tabela 2.

Tabela 2. Intervalos das classes utilizados no P-Index

\begin{tabular}{lc}
\hline Classes & Variação \\
Nula & $0-0,1$ \\
Baixa & $0,2-0,5$ \\
Média & $0,6-12,0$ \\
Alta & $12,1-144,0$ \\
Muito Alta & $>144,1$ \\
\hline
\end{tabular}

Os procedimentos para obtenção do P-Index são descritos a seguir:

O fator perda de solo foi obtido através da utilização da Equação Universal de Perda de Solos (EUPS) (Wischmeier \& Smith, 1978) em um ambiente SIG. Com o uso destas duas ferramentas foi possível quantificar e espacializar as perdas de solo para as bacias contribuintes dos reservatórios.

A EUPS é definida pela seguinte expressão:

$$
\mathrm{A}=\mathrm{R} \cdot \mathrm{K} \cdot \mathrm{LS} \cdot \mathrm{C} \cdot \mathrm{P}
$$

onde:

$$
\text { A - estimativa da perda de solo por erosão hídrica, }
$$
em $t$ ha $^{-1}$ ano $^{-1}$
$\mathrm{R}$ - erosividade da chuva, MJ mm ha-1 $\mathrm{h}^{-1} \mathrm{ano}^{-1}$

$\mathrm{K}$ - erodibilidade do solo, $\mathrm{t}$ ha $\mathrm{h} \mathrm{MJ}^{-1} \mathrm{~mm}^{-1} \mathrm{ha}^{-1}$

$\mathrm{L}$ - fator comprimento da rampa, adimensional

$\mathrm{S}$ - fator declividade, adimensional

C - fator uso e manejo do solo, adimensional

$\mathrm{P}$ - fator práticas conservacionistas, adimensional

$\mathrm{O}$ valor de $\mathrm{R}$ foi obtido do trabalho de Scalabrin et al. (1994) que obtiveram um valor médio anual, no período de 1982 a 1991, de $5.565 \mathrm{MJ} \mathrm{mm} \mathrm{ha}^{-1} \mathrm{~h}^{-1}$ ano $^{-1}$ para uma área próxima aos reservatórios. Os meses do ano com chuvas mais erosivas foram janeiro e fevereiro. $\mathrm{O}$ fator $\mathrm{R}$ possui efeito conjugado com o fator C (uso e manejo do solo); assim, nos períodos de maior erosividade da chuva deve-se evitar a realização de trabalhos de preparo do solo, pelo risco de ocorrência de altas taxas de erosão hídrica do solo.

O fator K foi calculado pela equação proposta por Denardin et al. (1990) a partir das informações contidas em Brasil (1973). O valor K calculado para este trabalho foi de 0,0494 tha h $\mathrm{MJ}^{-1} \mathrm{~mm}^{-1} \mathrm{ha}^{-1}$ considerando-se que o solo predominante nas bacias contribuintes é uma associação de Cambissolo Húmico com Neossolo Litólico.

Calculou-se o fator LS da EUPS através do software USLE-2D, validado por Pante et al. (2002) para o cálculo do fator LS de bacias hidrográficas. O software USLE-2D calcula o fator LS a partir de um modelo numérico de elevação (MNE). Para este trabalho, o MNE foi obtido a partir da digitalização de curvas de nível espaçadas de 20 em 20 metros, de cartas topográficas de escala 1:50.000 do Serviço Geográfico do Exército. Com o uso do software USLE-2D, gerou-se um arquivo tipo raster do fator LS, processado em ambiente de SIG (Idrisi), o qual foi exportado no formato ASCII para o software Spring (1996).

$\mathrm{O}$ fator $\mathrm{C}$ foi determinado conforme informações contidas na Tabela 3, na qual se procurou associar, a cada uso da terra, um fator $\mathrm{C}$ correspondente. $\mathrm{O}$ uso da terra na bacia hidrográfica foi determinado utilizando-se imagens de satélite (TM - LANDSAT, bandas 3, 4 e 5) do ano de 2002, através de uma classificação supervisionada.

Tabela 3. Valores do fator $C$ atribuídos aos diferentes usos da terra utilizado na EUPS

\begin{tabular}{lc}
\hline Usos da Terra & Fator C \\
Água & $0,000^{*}$ \\
Campo natural & $0,005^{*}$ \\
Mata nativa densa & $0,001^{*}$ \\
Mata nativa rala & $0,002^{*}$ \\
Florestamento & $0,005^{*}$ \\
Agricultura & $0,750^{\star *}$ \\
Área urbana & $0,030^{*}$ \\
\hline * Fonte: Wischmeier \& Smith (1978)
\end{tabular}

** Fonte: calculado para culturas olerícolas (batata e alho)

Ao fator $\mathrm{P}$ atribui-se o valor 1 , considerando-se a não adoção de práticas conservacionistas em toda a bacia hidrográfica.

Para determinação das perdas de solo realizaram-se, no passo seguinte, cruzamentos dos diferentes planos de informação representados pelos fatores da equação na forma de 
matriz numérica, com resolução de 30 × 30 m, no software Spring-INPE. Com isto se obteve, através da linguagem LEGAL, um novo plano de informações com as estimativas de perdas de solo da bacia hidrográfica, classificadas em cinco classes.

Os teores de fósforo no solo foram obtidos através de análises de solos coletados nos diferentes usos da terra, de forma a representar as diferentes classes de uso encontradas na bacia. Realizou-se a amostragem de solo utilizando-se um trado de rosca obtendo-se em torno de 20 subamostras por amostra de solo, totalizando 16 amostras dos diferentes usos da terra, nas diferentes porções da vertente, na profundidade de 0 a $20 \mathrm{~cm}$; as subamostras foram homogeneizadas para diminuir a variabilidade espacial do fósforo. Para determinação da concentração de fósforo nas amostras utilizou-se o método Mehlich I (Tedesco et al., 1995) devido a sua alta correlação com o fósforo disponível no solo. Através da associação da matriz de uso da terra com os teores de fósforo encontrados em cada classe de uso, foi possível estimar o nível de fósforo para os diferentes usos da terra na bacia.

A distância das áreas à rede de drenagem foi determinada em ambiente SIG através da determinação da distância do centro de cada pixel, à drenagem mais próxima. Elaborou-se o plano de informação distância da drenagem de acordo com os valores sugeridos por Weld et al. (2002).

Os planos de informação de estimativa de perdas por erosão, teores de fósforo no solo e distância da drenagem, foram reclassificados de acordo com a Tabela 1, gerandose planos de informação com os respectivos fatores (com valores zero a oito) a serem utilizados no cálculo do P-Index.

Obteve-se o plano de informação do P-Index através da multiplicação dos planos de informação com os fatores para estimativa da perda de solo, distância da drenagem e teor de fósforo no solo. Este plano de informação, por sua vez, foi reclassificado conforme as classes apresentadas na Tabela 2, obtendo-se o mapa de risco de contaminação de fósforo da bacia hidrográfica.

\section{RESULTADOS E DISCUSSÃO}

Apresentam-se, na Tabela 4, os diferentes tipos de uso da terra encontrados na bacia contribuinte dos reservatórios do Sistema Salto. Verifica-se, então, que o uso é constituído, predominantemente, de áreas de campo (76,1\%), florestamento $(9,0 \%)$, mata nativa $(13,0 \%)$, agricultura $(1,4 \%)$ e área urbana (0,5\%). A distribuição espacial desses usos é apresentada na Figura 1. Em relação às atividades agrícolas na bacia contribuinte, predominam a pecuária de corte extensiva, a exploração florestal com Pinus (P. elliottii var. elliottii) e, em uma escala menor, a agricultura intensiva com culturas olerícolas, como batata (Solanum tuberosum) e alho (Allium sativum). O cultivo de culturas olerícolas é predominantemente realizado por agricultores arrendatários e o sistema de florestamento é explorado por grandes empresas.
Tabela 4. Usos da terra na bacia hidrográfica do Sistema Salto e respectivas áreas, em percentagem

\begin{tabular}{lc}
\hline Classe de uso & Área (\%) \\
Campo & 76,1 \\
Mata nativa (densa e rala) & 13,0 \\
Florestamento & 9,0 \\
Agricultura & 1,4 \\
Área urbana & 0,5 \\
\hline
\end{tabular}

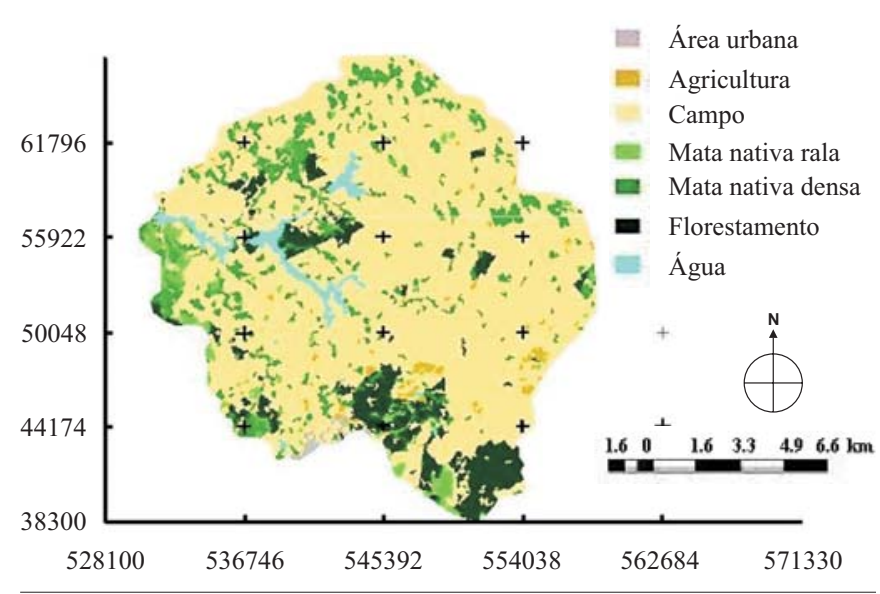

Figura 1. Uso da terra na bacia hidrográfica do Sistema Salto

Valores dos teores de fósforo no solo para as classes de uso campo, florestamento, mata nativa e agricultura foram respectivamente 2,6, 1,8, 2,6 e 20,6 $\mathrm{mg} \mathrm{kg}^{-1}$ verifica-se que, nas áreas de campo natural e florestamento, os níveis de fósforo no solo são considerados muito baixos, enquanto nas áreas de mata nativa esses níveis são aceitos como baixos; já nas áreas com agricultura, os teores de fósforo se encontram em níveis considerados altos, conforme os parâmetros de fertilidade do solo, definidos pela Rede de Laboratórios de Solos do Sul (SBCS, 2004), que interpreta os valores dos teores de fósforo no solo para as principais culturas do Rio Grande do Sul e de Santa Catarina, de acordo com a percentagem de argila de cada amostra coletada. No caso da mata nativa, campo e florestamento, os valores baixos de fósforo se justificam devido à condição de baixa fertilidade natural do solo e da não utilização de fertilizantes, tanto na pecuária extensiva como na implantação do cultivo de Pinus. Por outro lado, os elevados valores de fósforo encontrados nas áreas com agricultura decorrem das altas doses de fertilizantes utilizadas para as culturas olerícolas, em média $2000 \mathrm{~kg} \mathrm{ha}^{-1}$ da fórmula 20-20-20 (N-P-K). Essas aplicações são, muitas vezes, tidas como excessivas, pois não são reconhecidas as reais necessidades nutricionais das culturas nem a disponibilidade de nutrientes no solo; também foi possível observar, durante os levantamentos de campo, que as áreas sob agricultura apresentavam sérios problemas de degradação dos solos devido à erosão hídrica. A principal causa da ocorrência da erosão hídrica nessas áreas pode ser atribuída ao excessivo preparo de solo realizado no sentido do declive e da ausência de utilização de práticas de conservação do solo, por parte dos agricultores arrendatários (Cybis, 2005). 
Em relação às perdas de solo verifica-se que, na maior parte da área da bacia, elas são consideradas baixas, condição esta justificada pelo uso da terra predominante na bacia, a pecuária extensiva sobre vegetação de campo natural (Figura 2), que possibilita uma cobertura vegetal permanente. As áreas em que os valores de perda de solo são mais elevados coincidem com as áreas utilizadas com lavouras anuais e confirmam as observações de campo.

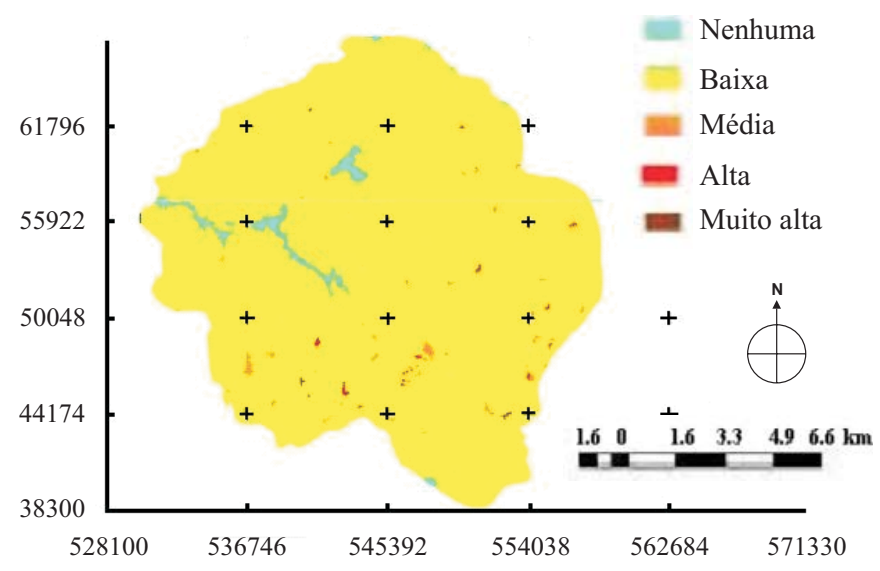

Figura 2. Perdas de solo na bacia hidrográfica do Sistema Salto

A combinação dos planos de informações referentes às perdas de solo, aos teores de fósforo no solo e à distância dessas áreas ao sistema de drenagem, resultou na elaboração do mapa da Figura 3, que representa a distribuição espacial do P-Index. A partir desta figura se verifica que a maior parte da área da bacia apresenta baixo risco de contaminação por fósforo, fato este explicado devido a maior parte da área da bacia ser ainda constituída por campo, onde a exploração com pecuária extensiva é praticada sem adição de fertilizantes nas pastagens sobre solos naturalmente pobres em fósforo. Por outro lado, as áreas de maior risco de contaminação por fósforo estão associadas àquelas áreas ocupadas com cultivos anuais que recebem grandes quantidades de fertilizantes e estão, em sua maioria, sendo manejadas de forma inadequada em virtude do preparo

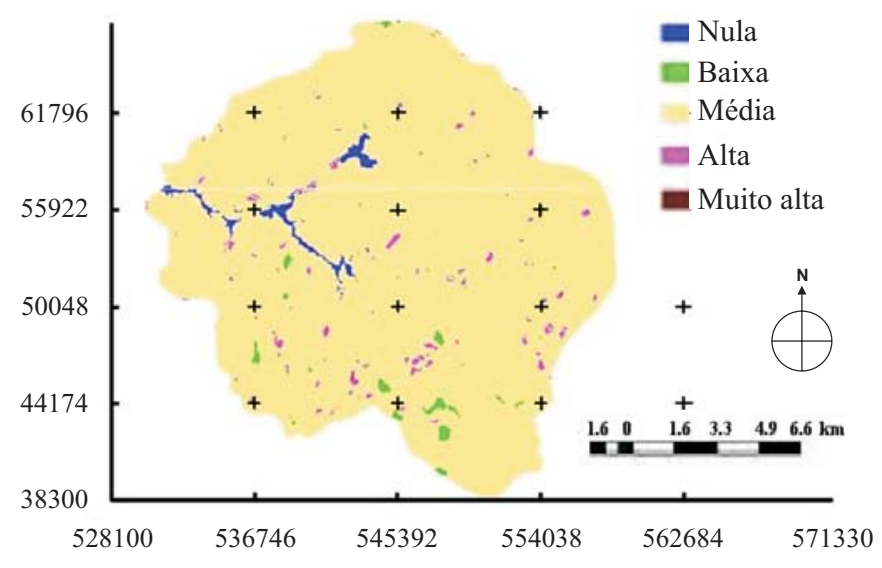

Figura 3. Risco potencial de contaminação por fósforo na bacia hidrográfica do Sistema Salto excessivo do solo e da não utilização de práticas conservacionistas.

Os resultados obtidos com a aplicação do P-Index mostram que a baixa intensidade de uso a que está sendo submetida a bacia hidrográfica do Sistema Salto, tem resultado em um baixo aporte de fósforo, com reflexos positivos sobre a qualidade da água nos reservatórios.

Os resultados apresentam consistência com dados do monitoramento da qualidade da água do Sistema Salto, o que indicou baixas concentrações de nutrientes nas águas caracterizando ambientes oligotróficos a mesotróficos. Ambientes oligotróficos caracterizam-se por baixas concentrações de um ou mais nutrientes que limitam a produtividade primária, enquanto os mesotróficos atingem um grau intermediário de trofia do ambiente aquático. O estado oligotrófico, por sua vez, sugere que a exportação de fósforo da bacia contribuinte para dentro dos reservatórios seja ainda baixa. Adicionalmente à qualidade da água dos reservatórios, os sedimentos do leito de alguns arroios tributários aos reservatórios também indicaram baixas concentrações de fósforo e comprovaram a condição de baixa disponibilidade de fósforo encontrada nos solos da bacia de contribuição (Franzen et al., 2004).

Ainda que o cenário atual do uso da terra na bacia estudada se tenha mostrado adequado para manter a qualidade de água dos reservatórios, verificam-se mudanças de uso, em função das condições econômicas desfavoráveis à pecuária extensiva, que representa a matriz produtiva principal da região dos reservatórios (Cybis, 2005). Os pecuaristas, em busca de uma renda maior, são estimulados a arrendar suas terras para agricultura, em especial para cultivos com espécies olerícolas de grande valor econômico; no entanto, essas culturas são altamente demandadoras de fertilizantes e agrotóxicos e, devido à ausência de adoção de práticas conservacionistas por parte dos agricultores arrendatários, essas áreas são consideradas de grande impacto à qualidade de água dos reservatórios. Uma segunda tendência de mudança de uso da terra da bacia, também provocada por razões econômicas, está ocorrendo em direção a uma expansão das áreas com florestamento com Pinus. Este tipo de uso, porém, oferece menores riscos à qualidade da água dos reservatórios mas pode causar impacto às condições hidrológicas da bacia, especialmente na condição em que o florestamento é implantado sobre áreas de nascentes. Já em relação ao P-Index utilizado neste trabalho, verificou-se ser uma ferramenta adequada para identificar áreas com potencial de contribuição de fósforo para os corpos d’água; entretanto, este modelo pode ser aperfeiçoado sempre que forem introduzidos mais planos de informações que possibilitem identificar, de forma ainda mais adequada, as fontes de fósforo para os ecossistemas aquáticos; um exemplo poderia ser a inclusão de um plano de informação que descrevesse as áreas consideradas hidrologicamente sensíveis, ou seja, as áreas preferenciais de formação do escoamento, conforme sugerem Gburek \& Sharpley (1998), potência do escoamento (Rose, 2004) e a presença de ambiente ciliar na calha do rio. 


\section{CONCLUSÕES}

1. De acordo com a aplicação do P-Index verificou-se para a bacia contribuinte do Sistema Salto, que a maior parte da bacia foi classificada como tendo baixo risco de contribuição de fósforo em decorrência do tipo de uso da terra predominante na bacia, campos explorados com pecuária extensiva.

2. As áreas de risco mais elevado foram identificadas como aquelas cujos solos são utilizados de forma mais intensiva com culturas anuais e sem a adoção de práticas conservacionistas.

3. A aplicação do P-Index apresenta um bom potencial para ser utilizado como instrumento de gestão dos recursos hídricos, contribuindo para a identificação de áreas de risco de poluição difusa e possibilitando, com isto, o planejamento mais eficiente das ações a serem realizadas para melhoria da qualidade dos recursos hídricos.

\section{LITERATURA CITADA}

Brasil. Ministério da Agricultura. Levantamento de reconhecimento dos solos do estado do Rio Grande do Sul. Recife: convênio MA/DPP - SA/DRNR, 1973. 431p. Boletim Técnico, 30.

Carmichael, W. W.; Azevedo, M. F. O.; An, J. S.; Molica, R. J. R.; Jochimsen, E. M.; Lau, S.; Rinehart, K. L.; Shaw, G. R.; Eagelsham, G. K. Human fatalities from cyanobacteria: Chemical and biological evidence for cyanotoxins. Environmental Health Perspectives, Cary, v.109, n.7, p.63-668, 2001.

Cybis, L. F. de A. Monitoramento ambiental e gestão integrada e sustentável dos reservatórios do Sistema Salto de Hidrelétricas (RS), com vistas à preservação da qualidade da água para abastecimento humano. Porto Alegre: IPH/UFRGS. 2005. 162p. Relatório Científico

Denardin, J. E. Erodibilidade do solo estimada através de parâmetros físicos e químicos. Piracicaba: ESALQIUSP, 1990. 113p. Tese Doutorado

Dils, R. M.; Heathwaite, A. L. Phosphorus fraction in hillslope hydrological pathways contributing to agricultural runoff. In: Anderson, M.G.; Brooks, S. M. (ed.). Advances in hillslope processes. Chichester: John Wiley \& Sons, 1996. cap.10, p.229-251.

Eghball, B.; Gilley, J. E. Phosphorus risk assessment index evaluation using runoff measurements. Journal of Soil and Water Conservation, v.56, n.3. p.202-206, 2001.

Franzen, M.; Cybis, L. F. A.; Merten, G. H.; Carvalho, E. N. Possíveis causas de disponibilização de fósforo nos reservatórios do sistema salto (RS). In: Simpósio Ecologia de Reservatórios, 2004, Avaré. Resumos... Avaré: Instituto de Biociências, UNESP, 2004. p.63.
Gburek, W. J.; Sharpley, A. N. Hydrologic controls on phosphorus loss from upland agricultural watersheds. Journal of Environmental Quality, Madison, v.2, n.27, p.267-277, 1998.

Lemunyon, J. L.; Gilbert, R. G. The concept and need for a phosphorus assessment tool. Journal of Production Agriculture, Madison, v.4, n.6, p.483-486, 1993.

Margalef, R. Limnologia. Barcelona: Editorial Omega. 1983. 1009p.

Pante, A.; Minella, J. P. G; Merten, G. H. Cálculo do fator LS da EUPS utilizando um método computacional. In: Reunião Brasileira de Manejo e Conservação do Solo e da Água, 14, 2002, Cuiabá. Resumos...Cuiabá: SBCS, 2002. p.153-166.

Rose, C. Na Introdution to the environmental physics of soil, water and watersheds. 1.ed. Cambridge: Cambridge University Press, 2004. 440p.

SBCS - Sociedade Brasileira de Ciência do Solo. Comissão de Química e Fertilidade do Solo - RS/SC. Manual de adubação e calagem para o Estado do Rio Grande do Sul e Santa Catarina. Porto Alegre: SBCS-NRS, 2004. 400p.

Scalabrin, G. A.; Silva, P. R.; Cassol, E. A. Erosividade das chuvas em Encruzilhada do Sul, Vacaria e Uruguaiana, RS, para um período de dez anos (1982-1991). In: Salão de Iniciação Científica, 6, 1994. Resumos...Porto Alegre: UFRGS, 1994. p.92.

Spring - Integrating remote sensing and GIS by object-oriented data modeling. Computers e Graphics, New York, v.20, n.3, p.395-403, 1996

Streck, E. V.; Kämpf, N.; Dalmolin, R. S. D.; Klamt, E.; Nascimento, P. C. do; Schneider, P. Solos do Rio Grande do Sul. 1.ed. Porto Alegre: EMATER/RS; UFRGS, 2002 107p.

Tedesco, M. J.; Gianello, C.; Bissani, C. A. Análises de solo, planta e outros materiais. 2.ed. Porto Alegre: Departamento de Solos/UFRGS, 1995. 174p. Boletim Técnico de Solos, 5

Tundisi, J. G.; Matsumura-Tundisi, T.; Arantes Júnior, J. D.; Tundisi, J. E. M.; Manzini, N. F.; Ducrot, R. The response of Carlos Botelho (Lobo, Broa) reservoir to the passage of cold fronts as reflected by physical, chemical, and biological variables. Brazilian Journal of Biology, São Carlos, v.64, n.1, p.177-186, 2004.

Vighi, M.; Chiaudani, G. Eutrophication in Europe: the role of agricultural activities. Hodgson, E. (ed.). Reviews in environmental toxicology 3. Amsterdam: Elsevier, 1987, p.213-258.

Weld, J. L.; Parkons, R. L.; Beegle, D. B.; Sharpley, A. N.; Gdurek, W. J; Clouser, W. R. Evaluation of phosphorus - based nutrient management strategies in Pennsylvania. Journal of Soil and Water Conservation, Ankeny, v.57, n.6, p.448-454, 2002.

Wischmeier, W. H.; Smith, D. D. Predicting rainfall erosion losses - a guide to conservation planning. USDA Agriculture Handbook. U.S. Washington, DC: Government Printing Office, 1978.58p.

Yoo, R. S.; Carmichael, R. C.; Hoehn, R. C.; Hrudey, S. E. Cyanobacterial (blue-green algal) toxins: a resource guide. AWWA Research Foundation, Denver, 1995. 229p. 